\title{
Improving sensitivity of amyloid detection by Congo red stain by using polarizing microscope and avoiding pitfalls
}

\author{
Ashraf El-Meanawy $^{1^{*}}$ (D) Christopher Mueller ${ }^{1}$ and Kenneth A. Iczkowski ${ }^{2}$
}

\begin{abstract}
Systemic amyloidosis is a devastating group of disorders for which there is no current cure. The treatment goal is to reduce the burden of amyloidogenic protein precursors. The treatment is only effective if applied early in the disease process before significant and irreversible end organ damage has taken place. Congo red is still the standard stain used in most histopathology laboratories to identify amyloid material in tissues. The identification of Congophilic amyloid material is challenging because of multiple interfering factors. Here we describe improved sensitivity of identifying Congophilic materials in histologic sections using a metallurgical polarized microscope specifically constructed for polarized microscopy. The microscope is equipped with strain-free optics, matching polarizers, dis-integrated compensators, and a circular mobile stage. Compared to a standard clinical microscope, this setup significantly improves sensitivity of identifying amyloid material in Congo red-stained slides. We also describe the deleterious effect of plastic coverslip which can interfere with the ability to examine the slides under polarized light. We present a series of 10 different patients who had cardiac, brain, and salivary gland biopsies that were either equivocal or deemed negative using a standard clinical microscope but were positive using the equipment described above. These samples were confirmed to be positive by other methods including electron microscopy. We conclude that use of the correct equipment is needed before ruling out amyloidosis in tissue sections.
\end{abstract}

Keywords: Amyloidosis, Metallurgical microscope, Polarized microscopy, Congo red

\section{Background}

Amyloidosis is a group of disorders caused by protein misfolding and aggregation. Systemic amyloidosis is a consequence of circulating amyloidogenic protein monomers which deposit in various tissues with variable affinities, causing tissue damage and multi-organ dysfunction. The term amyloid is a misnomer based on Rudolf Virchow's mistakenly identifying the material as starch (Amylin) [1]. More than 40 different proteins have been identified as a precursor for amyloid formation in humans [2]. The most common forms of systemic amyloid are $\mathrm{AL}$ amyloid seen in plasma cell dyscrasias, AA amyloid associated with inflammatory conditions, and TTR amyloidosis due to either familial gene mutation or wild type protein,

\footnotetext{
* Correspondence: renalresearch@gmail.com

'Department of Medicine, Medical College of Wisconsin, 8701 Watertown

Plank Rd, Milwaukee, WI 53226, USA

Full list of author information is available at the end of the article
}

formerly called senile amyloidosis. The clinical picture and prognosis of amyloidosis is dependent on organ(s) affected and whether organ dysfunction can be identified by symptoms or quantified by functional testing, particularly heart, kidney, and nervous system which are frequently involved and trigger testing.

Cardiac involvement is manifest in approximately one-third to one-half of all AL patients at the time of diagnosis [3]. The predominant presenting symptom is rapidly progressive heart failure with preserved ejection fraction. Unfortunately, symptoms of cardiac decompensation are also a major risk factor for mortality. Kyle et al. showed that in 168 patients with systemic amyloidosis, those who presented with congestive heart failure had a median survival of 4 months [4]. Renal amyloidosis is frequently, but not necessarily always, associated with high grade proteinuria. The trigger for amyloid workup is usually nephrotic syndrome and/or decline of renal function. Renal amyloidosis 
is not as rare as it was once thought to be. Retrospective analysis of 17 years of renal biopsies in the Czech Republic revealed that $43 \%$ of cases of nephrotic syndrome above the age of 60 were due to amyloidosis [5]. Neuropathy is another significant manifestation of neuron-avid monomers, which can be devastating leading to peripheral and/or autonomic neuropathies with crippling manifestations [6, 7].

The frequently observed rapid functional recovery of kidney and heart with therapies that limit or abolish monomer production is often attributed to direct monomer and/or oligomer toxicity $[8,9]$. But, it also underscores the imperative of establishing the diagnosis of amyloid as early as possible so that effective therapy can be instituted. Unfortunately, the diagnosis of amyloid in tissue sections is challenging. Identification of fibrils by electron microscopy (EM) is highly specific but because of the patchy nature of the disease, and the magnification level of EM, the sensitivity is very poor a region of interest can be identified using another method. Thioflavin-T is very sensitive $[10,11]$ but its specificity is not trusted by some experts. Interpretation of Thioflavin-T staining is also plagued by its tendency to bleach, as well as the subjective assessment of intensity based on comparison to a very strong positive control. Congo red, despite having lower sensitivity, is the standard agent used to identify amyloid in tissues. While the apple-green birefringence seen under crossed polarized light is specific for amyloid material, staining with Congo red is technically difficult resulting in inconsistent staining. Moreover, variation of mounting media and limitations of the examining microscopes increase both false-negative and false-positive results. Pitfalls of the staining techniques have been addressed elsewhere [12-14]. In this paper we show that the use of a microscope built specifically for polarized light increases sensitivity of identifying amyloid in Congo red stained sections. We also describe minor pitfalls in examination of Congo red stained slides.

\section{Methods}

The metallurgical microscope used in the current report is built specifically for polarized microscopy. These microscopes are widely available from different manufactures and cost between $\$ 13,000-\$ 20,000$ which is comparable to standard clinical microscopes. The microscope is equipped with strain free objectives, condenser, and eyepieces. The strain free optics is critical to eliminate the false optical effects generated by stressed glass under polarized light. These spurious artifacts can interfere with the ability to evaluate birefringence produced by Congo red stained amyloid deposits. The condenser, besides having strain free lenses, is designed to produce perfect parallel beam of light. One of the most important features of a polarized microscope is the $360^{\circ}$ circular rotating stage. This allow easy and full rotation of each examined field in the specimen. The microscopes we used have a fixed polarizer fitted in the condenser and an analyzer with a precise degree dial for cross setup. The illustration accompanying the test is generic and can apply to any polarized microscope (Microscope illustration).

Such microscope is ideal for polarized microscopy and is superior to a clinical microscope for examining Congo red stained sections. We obtained similar results using similar microscopes from Leica and Nikon. Using Sénarmont compensator did not provide added benefit. The same slides were also examined using a clinical microscope in which the analyzer has a built-in $\lambda$ compensator, which is common equipment sold with clinical microscopes. The $\lambda$ compensators are useful in examining crystals such as uric acid but can hamper detection of amyloid deposits. All the polarized microscopes were equipped with a circular rotating mechanical stage.

We specifically selected cardiac, salivary gland, and brain biopsies, which were initially deemed negative when examined by clinical microscope but were later confirmed positive for amyloidosis. These cases cover 3 types of amyloid AL, ATTR, and FGA (Table 1). Regardless of the amyloid type, the polarized microscope was superior to clinical microscope. Commercially purchased positive controls were also examined to demonstrate the validity of the techniques. Although Congo staining technique vary from lab to lab, we found no significant difference between in-house prepared stain, vs Leica, vs Dayko stains

\section{Results}

We found that the apple green birefringence is more readily visible and with higher intensity when the slides are examined using a metallurgical microscope compared to the standard clinical microscope. Fig. 1 shows an image of biopsies examined by the metallurgical microscope for comparison purpose, the representative same field imaged with a standard microscope is shown. Additional file 1 has a series of biopsy samples examined by clinical and metallurgical microscope. As it is known that Congo red can be fluorescent, we examined the slides with fluorescent microscope using Texas red filter. Figure 2 shows representative image displaying the red fluorescence of Congo red in tissues. Fluorescence is more sensitive but less specific for amyloid stained with Congo red [15].

\section{A plastic coverslip can obliterate the ability to examine slides properly}

We examined 2 samples (kidney and heart) from 2 different institutions where plastic cover slips were used. 
Table 1 List of reviewed cases, tissue examined, and amyloid type

\begin{tabular}{c|c|c}
\hline \multicolumn{2}{c|}{ Examined Tissue } & MASS-SPEC Amyloid type \\
\hline 1 & Heart & ATTR \\
\hline 2 & Heart & ATTR \\
\hline 3 & Heart & ATTR \\
\hline 4 & Heart & $\mathrm{AL}$ \\
\hline 5 & Heart & $\mathrm{AL}$ \\
\hline 6 & Heart & $\mathrm{AL}$ \\
\hline 7 & Heart & $\mathrm{AL}$ \\
\hline 8 & Heart & Not typed \\
\hline 9 & Brain and kidney & $\mathrm{FGA}$ \\
\hline 10 & Salivary gland & $\mathrm{AL}$ \\
\hline
\end{tabular}

We found that plastic coverslips scatter light and inconsistently polarize in a pattern that makes it impossible to cross the microscope polarizer and analyzer properly to obtain a dark field. Figure 3a shows a comparison of the light passed through a slide with plastic cover slip at the edge of the cover. The plastic coverslip allowed the light to go through while glass did not. This prevents examining the slide under crossed polarized light. Unfortunately, the outside labs exhausted both biopsy tissues from these patients and it was not possible to procure more material. To further examine the effect of plastic coverslips we obtain cover slips from 2
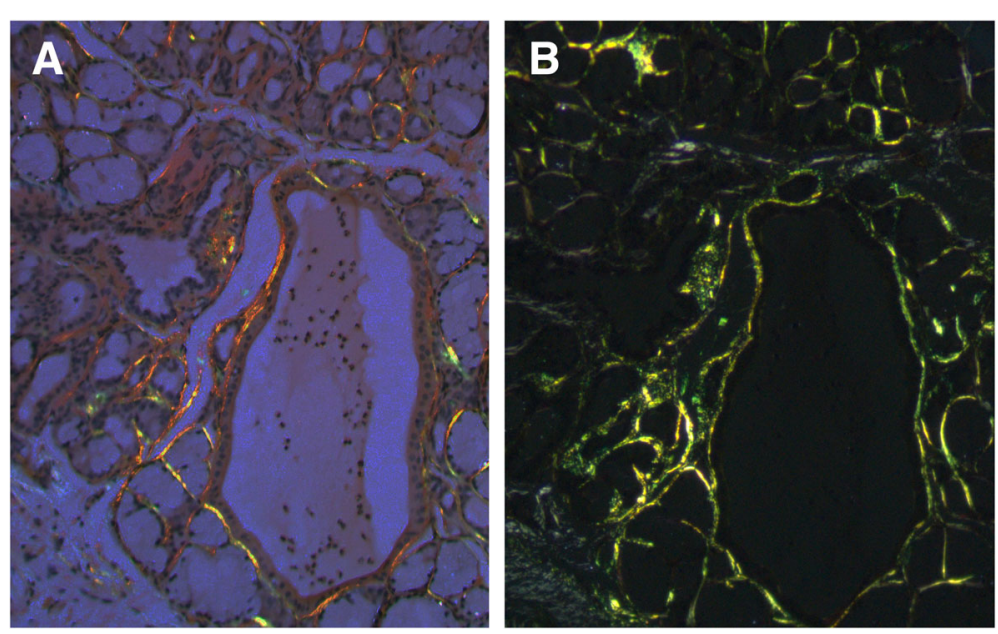

Fig. 1 Congo red stained salivary gland section examined under crossed polarized light from patient with AL amyloid imaged using clinical microscope (A) and same field examined using Metallurgical microscope 


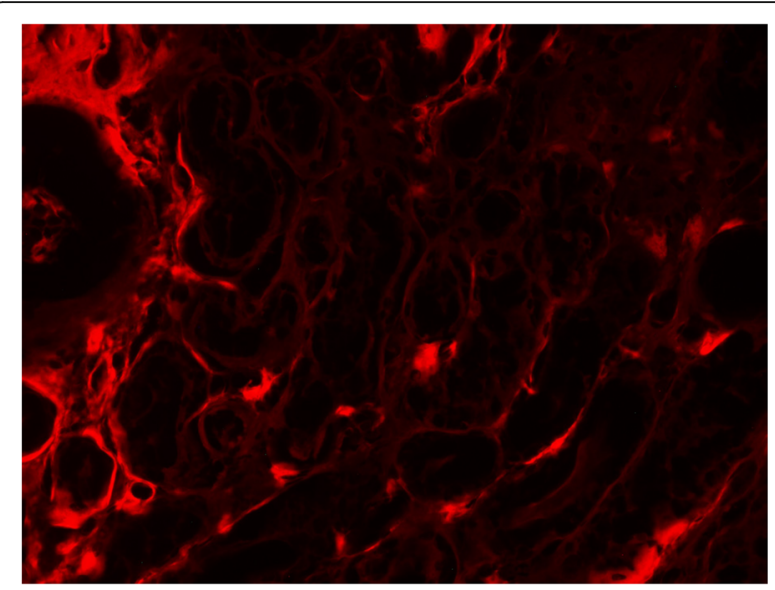

Fig. 2 Congo red examined with fluorescent microscope using a Texas-red filter

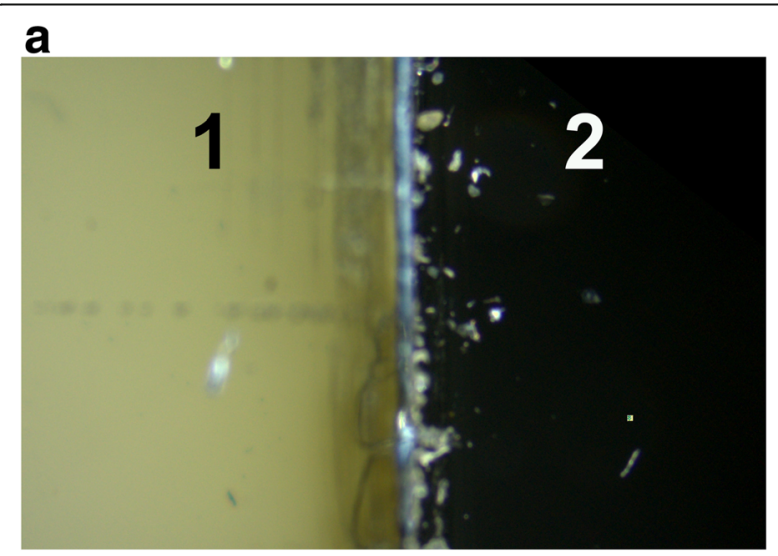

b

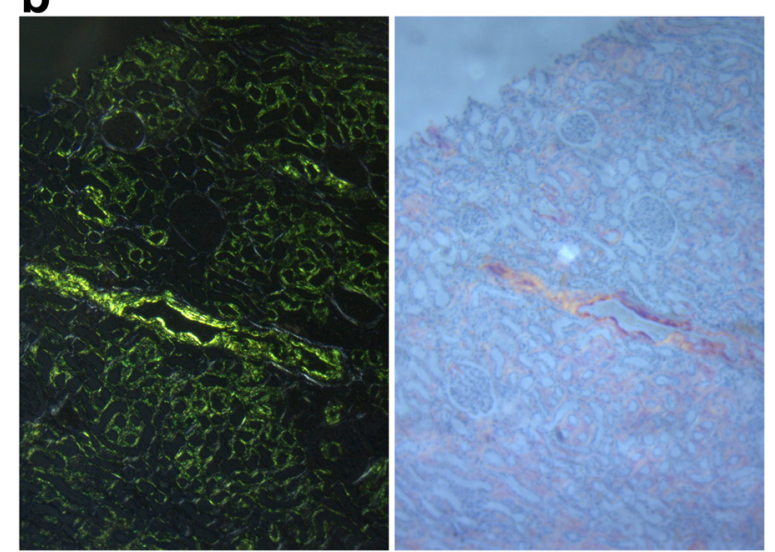

Fig. 3 a Negative impact of plastic coverslips in polarized microscopy, due to disorganized polarizing effect of some plastic polymers, it is difficult to obtain proper crossing of polarized light and light pass through on cover slip side (1) and proper dark field without the coverslip (2). b Negative impact of plastic cover slip in polarized microscopy showing positive control sample examined with glass cover slip (left) or plastic coverslip (right) different manufacturers. Both have ill effect on examining Congo red stained sections. Figure 3b showed positive control viewed through glass and plastic coverslips. We also observed that plastic effect was different depending on the manufacturer and as shown when rotating the slides. Video recording of rotated slides covered with glass or plastic coverslips is seen in Additional file 1.

\section{Rotating slides using a circular stage is important}

The orientation of the Congo red stained amyloid fibrils in relation to the plane of the light path can alter detection. Figure 4 shows samples imaged, then re-imaged after rotating the stage. It is evident that the apple green birefringence can be seen only at one angle. This highlights the need to use a mechanical circular stage, not found on clinical microscopes.

\section{Blue hue}

A common clinical analyzer has a polarizing filter with a built-in compensator which usually adds color and is often used for crystal examination. The use of such an analyzer in examining Congo red stained

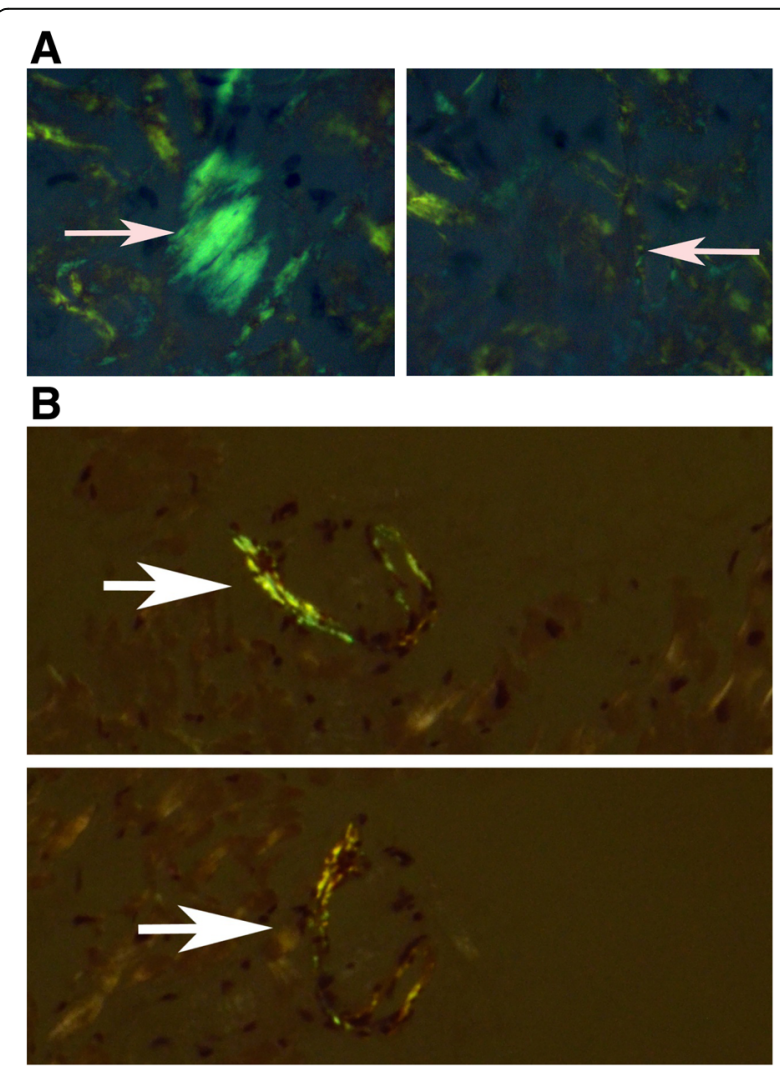

Fig. 4 shows a sample imaged then re-images after rotating the stage. $4 \mathrm{~A}$ rotated 45 degrees and $4 \mathrm{~b}$ rotated 60 degrees. The green birefringence is no longer visible as indicated by the arrows 
specimens, frequently leads to a field that is excessively bright and sometimes forces the observer to partially uncross the analyzer in order to accentuate the apple green birefringence. However, this results in appearance or enhancement of a bluish-green hue (Fig. 5) mainly due to collagen and other matrix proteins $[16,17]$.

\section{Discussion}

In this report, we show that suitable microscopy equipment can increase the sensitivity of identifying the amyloid-specific birefringence in Congo red-stained tissue sections. Early diagnosis of systemic amyloidosis is essential to reducing morbidity and mortality of the disease. Despite the seriousness of the disease and the benefit of early detection, an accurate pathologic diagnosis is still challenging. Spotty nature of disease, variation in organ-to-organ of the density of amyloid deposits, and the difficult of reproducible tissue staining, all increase the odds of false negative and false positive results. A negative tissue pathology report can effectively exclude the diagnosis of amyloidosis, which is then frequently never reconsidered among differential.

The standard of care for multiple myeloma, mono, and polyclonal gammopathy is conservative follow up, unless there is identifiable end-organ damage or amyloidosis [18]. Accordingly, missing an early diagnosis of amyloidosis can deprive a patient from receiving lifesaving treatment and can lead to costly and sometimes invasive investigations to pursue alternative diagnoses. In the case of transthyretin or fibrinogen amyloidosis, early liver transplantation usually arrests disease progression and can even be curative [19-21]. Therefore,

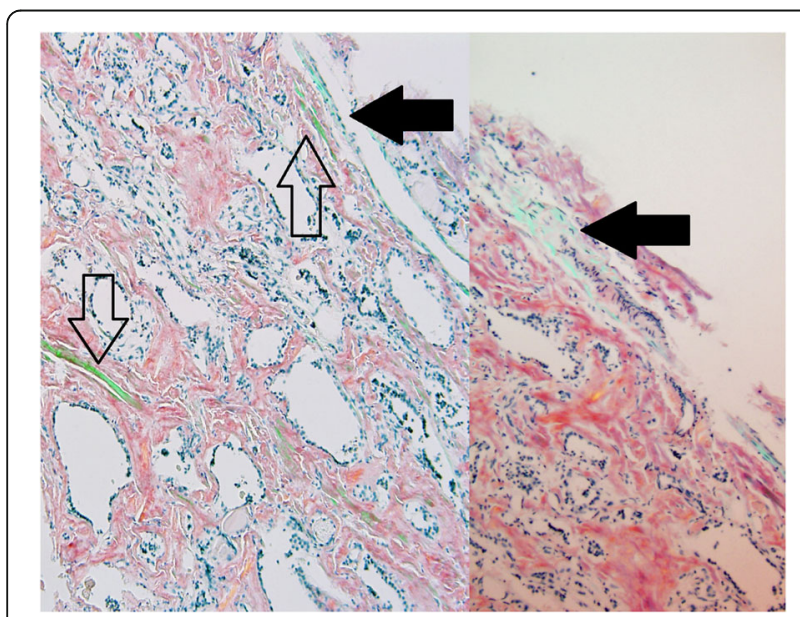

Fig. 5 Congo red stained slide images using standard microscope with an analyzer that has a build-in compensator. Blue hue (black arrows) compared to apple green (frame arrows). Sometimes this is caused by partial uncrossing of an analyzer with built in compensator even a marginal improvement in sensitivity of detection of amyloid in tissue specimens will help in assuring that patients with this serious and frequently fatal disease can be treated promptly and receive accurate prognostic information.

The real prevalence of amyloidosis is not known. A retrospective evaluation of kidney biopsies suggests that amyloidosis is not as rare as it is thought to be accounting to $43 \%$ of nephrotic proteinuria above age of 60 [5].

In single center experience $31 \%$ of patients with multiple myeloma patients has had confirmed evidence of amyloidosis [22]. The prevalence of multiple myeloma is dwarfed by the prevalence of monoclonal gammopathy, which can be as high as $8.4 \%$ depending on the race [23-25].

When the diagnosis is missed discipline-specific bias leads to ascribing to organ dysfunction to diabetes in case of renal disease and neuropathy and cardiac symptoms on hypertension or ischemia. Yet, there is no systematic data that examine the accuracy of these presumptive etiologies, and it is not inconceivable that some fraction of these patients may be incorrectly classified.

Owing to the patchy nature of amyloidosis, especially during its early stages, amyloid deposition could be restricted to just a small area of the tissue biopsy only visible at a limited angle of slide viewing. Thorough examination of each section using a mechanical rotating stage to view slides at variable angles is essential to avoid missing such deposits. We also recommend that plastic cover slips be avoided as they can interfere with the ability to perform crossed polarized light examination and reduce ability to identify subtle or low density amyloid deposits. Low density deposits are enough to make the diagnosis due to the patchy nature of the disease. When a sample is deemed negative or equivocal, there is a need to follow previous published modifications like the use of polar mounting media or omitting the alcohol differentiation step when examining collagen rich tissue to avoid interference [26-29]. Finally, the use of proper optics like those of a metallurgical microscope is essential to avoid missing the presence of small deposits of amyloid in Congo red-stained tissue.

\section{Conclusions}

There is variability on the reporting of Congo red stained slides between different labs and pathologists. We identified important pearls that can improve the ability to identify amyloid material in Congo red stained tissues. We found that it is critical to use microscope with proper strain free optics and avoid the use of polarizer with built-in compensator. The use of mechanical rotating stage will reduce the chance of missing 
subtle or low-level amyloid deposits which can only produce birefringence at specific angles. Last, plastic cover slips can lead to inability to examine the slides under crossed polarized light. Improving sensitivity of the Congo red evaluation can aid in early diagnosis of amyloid and will have tremendous impact on clinical outcome of some patients.

\section{Additional file}

Additional file 1: Included a video recording of congored stained tissue covered by eith glass or plastic coverslips and rotated. (PPTX $11971 \mathrm{~kb})$

\section{Abbreviation \\ AA: Amyloid-A; AL: Amyloid Light chain; EM: Electron microscopy; TTR: Transthyretin}

\section{Acknowledgements}

We would like to thank Zeiss USA, Leica USA, and Nikkon USA for allow us to compare their polarized microscopes.

\section{Funding}

No funding. Any additional cost was incurred by the submitting author personal funds.

\section{Availability of data and materials}

Supplemental data included.

\section{Authors' contributions}

AE carried out design, microscope testing, image capture, image comparison, drafting manuscript, and figures generation. CM contributed cardiac amyloid patient information and manuscript editing. Kl contributed false negative rate alert that triggered the work, clinical microscopy image capture, image comparison evaluation, and manuscript editing. All authors read and approved the final manuscript.

\section{Ethics approval and consent to participate}

$N A$, all the samples used were part of standard clinical care of subjects.

\section{Consent for publication}

NA

\section{Competing interests}

The authors declare that they have no competing interests.

\section{Publisher's Note}

Springer Nature remains neutral with regard to jurisdictional claims in published maps and institutional affiliations.

\section{Author details}

'Department of Medicine, Medical College of Wisconsin, 8701 Watertown Plank Rd, Milwaukee, WI 53226, USA. ${ }^{2}$ Departments of Pathology, Medical College of Wisconsin, Milwaukee, WI, USA.

Received: 20 December 2018 Accepted: 10 May 2019 Published online: 14 June 2019

\section{References}

1. Kyle RA. Amyloidosis: a convoluted story. Br J Haematol. 2001;114(3):529-38.

2. Sipe JD, Benson MD, Buxbaum JN, Si I, Merlini G, Saraiva MJM, et al. Amyloid fibril proteins and amyloidosis: chemical identification and clinical classification International Society of Amyloidosis 2016 nomenclature guidelines. Amyloid. 2016;23(4):209-13.

3. Esplin BL, Gertz MA. Current trends in diagnosis and Management of Cardiac Amyloidosis. Curr Probl Cardiol. 2013;38(2):53-96.

4. Kyle RA, Greipp PR, Fallon WM. Primary systemic amyloidosis: multivariate analysis for prognostic factors in 168 cases. Blood. 1986;68(1):220.
5. Maixnerova D, Jancova E, Skibova J, Rysava R, Rychlik I, Viklicky O, et al. Nationwide biopsy survey of renal diseases in the Czech Republic during the years 1994-2011. J Nephrol. 2014.

6. Shin SC, Robinson-Papp J. Amyloid neuropathies. Mt Sinai J Med. 2012;79(6):733-48.

7. Adams D, Lozeron P, Lacroix C. Amyloid neuropathies. Curr Opin Neurol. 2012;25(5):564-72

8. Tamaki H, Naito Y, Lee-Kawabata M, Taniguchi $Y$, Hao H, Hirota S, et al. Sustained improvement in cardiac function with persistent amyloid deposition in a patient with multiple myeloma-associated cardiac amyloidosis treated with bortezomib. Int J Hematol. 2010;92(4):655-8.

9. Decourt A, Gondouin B, Delaroziere JC, Brunet P. Sall+ᄀe M, Burtey S, et al. trends in survival and renal recovery in patients with multiple myeloma or light-chain amyloidosis on chronic dialysis. Clin J Am Soc Nephrol. 2016:11(3):431-41.

10. Novak L, Cook WJ, Herrera GA, Sanders PW. AL-amyloidosis is underdiagnosed in renal biopsies. Nephrol Dial Transplant. 2004:19(12):3050-3.

11. Herrera GA, Joseph L, Gu X, Hough A, Barlogie B. Renal pathologic spectrum in an autopsy series of patients with plasma cell dyscrasia. Arch Pathol Lab Med. 2004;128(8):875-9.

12. Bely M, Makovitzky J. Sensitivity and specificity of Congo red staining according to Romhànyi. Comparison with Puchtler's or Bennhold's methods. Acta Histochem. 2006;108(3):175-80.

13. Howie AJ, Brewer DB. Optical properties of amyloid stained by Congo red: history and mechanisms. Micron. 2009:40(3):285-301.

14. Makovitzky J, Richter S, Appel TR. Topooptical investigations and enzymatic digestions on tissue-isolated amyloid fibrils. Acta Histochem. 2006:108(3):193-6.

15. Linke RP. Diagnosis of minimal amyloid deposits using the Congo red fluorescence method. In: Picken MD PFMDMDPhDAHMDG, editor. Amyloid and Related Disorders. Current Clinical Pathology. Humana Press; 2012.

16. Shidham VB, Hunt B, Jaradeh SS, Barboi AC, Devata S, Hari P. Performing and processing FNA of anterior fat pad for amyloid. J Vis Exp. 2010:30(44):1747

17. Howie AJ. Apple-green birefringence? The bulletin of the Royal College of pathologists 2008:(144):263-266.

18. Bird J, Behrens J, Westin J, Turesson I, Drayson M, Beetham R, et al. UK myeloma forum (UKMF) and Nordic myeloma study group (NMSG): guidelines for the investigation of newly detected M-proteins and the management of monoclonal gammopathy of undetermined significance (MGUS). Br J Haematol. 2009;147(1):22-42.

19. Stangou AJ, Banner NR, Hendry BM, Rela M, Portmann B, Wendon J, et al. Hereditary fibrinogen a alpha-chain amyloidosis: phenotypic characterization of a systemic disease and the role of liver transplantation. Blood. 2010:115(15):2998-3007.

20. Gillmore JD, Booth DR, Rela M, Heaton ND, Rahman V, Stangou AJ, et al. Curative hepatorenal transplantation in systemic amyloidosis caused by the Glu526Val fibrinogen alpha-chain variant in an English family. QJM. 2000:93(5):269-75.

21. Pomfret EA, Lewis WD, Jenkins RL, Bergethon P, Dubrey SW, Reisinger J, et al. Effect of orthotopic liver transplantation on the progression of familial amyloidotic polyneuropathy. Transplantation. 1998;65(7):918-25.

22. Desikan KR, Dhodapkar MV, Hough A, Waldron T, Jagannath S, Siegel D, et al. Incidence and impact of light chain associated $(\mathrm{AL})$ amyloidosis on the prognosis of patients with multiple myeloma treated with autologous transplantation. Leukemia Lymphoma. 1997;27(3-4):315-9.

23. Cabrera Q, Macro M, Hebert B, Cornet E, Collignon A, Troussard X. Epidemiology of monoclonal Gammopathy of undetermined significance (MGUS): the experience from the specialized registry of hematologic malignancies of Basse-Normandie (France). Cancer Epidemiol. 2014;38(4):354-6

24. Agarwal A, Ghobrial IM. Monoclonal gammopathy of undetermined significance and smoldering multiple myeloma: a review of the current understanding of epidemiology, biology, risk stratification, and management of myeloma precursor disease. Clin Cancer Res. 2013; 19(5):985-94.

25. Wadhera RK, Rajkumar SV. Prevalence of monoclonal gammopathy of undetermined significance: a systematic review. Mayo Clin Proc. 2010; 85(10):933-42.

26. Romhanyi G. Selective differentiation between amyloid and connective tissue structures based on the collagen specific topo-optical staining 
reaction with Congo red. Virchows Arch A Pathol Pathol Anat. 1971; 354(3):209-22

27. Romhanyi G, Deak G, Bukovinszky A. Collagen-specific topo-optical staining reaction with Congo red and its ultrastructural interpretation. Acta Morphol Acad Sci Hung. 1970;18(3):261-82.

28. Elghetany MT, Saleem A, Barr K. The Congo red stain revisited. Ann Clin Lab Sci. 1989;19(3):190-5.

29. Bely M, Makovitzky J. Sensitivity and specificity of Congo red staining according to Romhanyi. Comparison with Puchtler's or Bennhold's methods. Acta Histochem. 2006;108(3):175-80.

Ready to submit your research? Choose BMC and benefit from:

- fast, convenient online submission

- thorough peer review by experienced researchers in your field

- rapid publication on acceptance

- support for research data, including large and complex data types

- gold Open Access which fosters wider collaboration and increased citations

- maximum visibility for your research: over $100 \mathrm{M}$ website views per year

At BMC, research is always in progress.

Learn more biomedcentral.com/submissions 